\title{
Circular RNA circCORO1C promotes laryngeal squamous cell carcinoma progression by modulating the let-7c-5p/ PBX3 axis
}

Yongyan $\mathrm{Wu}^{1,2,3,4,5+}$, Yuliang Zhang ${ }^{1,2+}$, Xiwang Zheng ${ }^{1,2+}$, Fengsheng Dai ${ }^{1,3+}$, Yan $\mathrm{Lu}^{6+}$, Li Dai ${ }^{1,3}$, Min Niu ${ }^{1,2}$,


Yu Zhang ${ }^{4,10}$, Tao Yang ${ }^{5}$, Li Li ${ }^{9}$, Linshi Zhang ${ }^{11}$, Rui Hou ${ }^{12}$, Shuxin Wen ${ }^{13}$, Changming An ${ }^{14^{*}}$, Huizheng Li ${ }^{15^{*}}$, Wei Xu ${ }^{16,17,18^{*}}$ and Wei Gao ${ }^{1,2,3,4,9^{*}}$ (D)

\begin{abstract}
Background: Laryngeal squamous cell carcinoma (LSCC) is a common malignant tumor of the head and neck. LSCC patients have seriously impaired vocal, respiratory, and swallowing functions with poor prognosis. Circular RNA (circRNA) has attracted great attention in cancer research. However, the expression patterns and roles of circRNAs in LSCC remain largely unknown.

Methods: RNA sequencing was performed on 57 pairs of LSCC and matched adjacent normal mucosa tissues to construct circRNA, miRNA, and mRNA expression profiles. RT-PCR, qPCR, Sanger sequencing, and FISH were undertaken to study the expression, localization, and clinical significance of cirCCORO1C in LSCC tissues and cells. The functions of circCORO1C in LSCC were investigated by RNAi-mediated knockdown, proliferation analysis, EdU staining, colony formation assay, Transwell assay, and apoptosis analysis. The regulatory mechanisms among circCORO1C, let-7c-5p, and PBX3 were investigated by luciferase assay, RNA immunoprecipitation, western blotting, and immunohistochemistry.
\end{abstract}

(Continued on next page)

\footnotetext{
*Correspondence: anchangming@cicams.ac.cn; huizhengli2004@163.com; xuwhns@126.com; gaoweisxent@sxent.org

†Yongyan Wu, Yuliang Zhang, Xiwang Zheng, Fengsheng Dai and Yan Lu contributed equally to this work.

${ }^{14}$ Department of Head and Neck Surgery, Cancer Hospital, National Cancer Center, Chinese Academy of Medical Sciences \& Peking Union Medical College, Beijing 100021, People's Republic of China

${ }^{15}$ Department of Otolaryngology Head \& Neck Surgery, Dalian Municipal Friendship Hospital, Dalian Medical University, Dalian 116100, Liaoning, People's Republic of China

${ }^{16}$ Shandong Provincial ENT Hospital Affiliated to Shandong University, Jinan 250022, Shandong, People's Republic of China

'Shanxi Key Laboratory of Otorhinolaryngology Head and Neck Cancer, Shanxi Medical University, Taiyuan 030001, Shanxi, People's Republic of China Full list of author information is available at the end of the article
}

(c) The Author(s). 2020 Open Access This article is licensed under a Creative Commons Attribution 4.0 International License, which permits use, sharing, adaptation, distribution and reproduction in any medium or format, as long as you give appropriate credit to the original author(s) and the source, provide a link to the Creative Commons licence, and indicate if changes were made. The images or other third party material in this article are included in the article's Creative Commons licence, unless indicated otherwise in a credit line to the material. If material is not included in the article's Creative Commons licence and your intended use is not permitted by statutory regulation or exceeds the permitted use, you will need to obtain permission directly from the copyright holder. To view a copy of this licence, visit http://creativecommons.org/licenses/by/4.0/ The Creative Commons Public Domain Dedication waiver (http://creativecommons.org/publicdomain/zero/1.0/) applies to the data made available in this article, unless otherwise stated in a credit line to the data. 
(Continued from previous page)

Results: circCORO1C was highly expressed in LSCC tissues and cells, and this high expression was closely associated with the malignant progression and poor prognosis of LSCC. Knockdown of circCORO1C inhibited the proliferation, migration, invasion, and in vivo tumorigenesis of LSCC cells. Mechanistic studies revealed that circCORO1C competitively bound to let-7c-5p and prevented it from decreasing the level of PBX3, which promoted the epithelial-mesenchymal transition and finally facilitated the malignant progression of LSCC.

Conclusions: circCORO1C has an oncogenic role in LSCC progression and may serve as a novel target for LSCC therapy. circCORO1C expression has the potential to serve as a novel diagnostic and prognostic biomarker for LSCC detection.

Keywords: circCORO1C, Let-7c-5p, PBX3, Laryngeal squamous cell carcinoma, Epithelial-mesenchymal transition, Metastasis

\section{Background}

Laryngeal squamous cell carcinoma (LSCC) is a common malignant tumor of the head and neck originating from the laryngeal mucosal epithelium. LSCC accounts for approximately $2.4 \%$ of systemic malignancies worldwide each year; in 2018, around 95,000 people died of laryngeal cancer [1]. The onset of LSCC is occult, and approximately $60 \%$ of patients are in the advanced stages when diagnosed (clinical stages III and IV) [2]. The proneness of LSCC to local invasion and cervical lymph node metastasis seriously interferes with patient survival rates [3]. Surgery remains the main treatment approach for LSCC [4]. Unfortunately, LSCC is one of the few tumors with a decreasing survival rate in recent years, and its 5-year survival rate has declined from 66 to $63 \%$ over the past 40 years [5], which is mainly attributed to its unclear mechanism of occurrence and progression. Therefore, it is urgent to reveal the pathogenesis of LSCC, identify biomarkers for its diagnosis, and investigate effective new therapeutic targets.

Circular RNA (circRNAs) is a recently identified noncoding RNA that has become the latest hotspot in cancer research. The circRNA molecule has a closed loop structure that is not affected by exonucleases and is not easily degraded. circRNAs also have features of high conservation and abundance [6]. Hence, circRNAs have unique advantages as biomarkers for disease diagnosis and prognosis. Recent studies have shown that circRNA molecules are rich in miRNA binding sites and can specifically bind miRNAs, thereby eliminating the inhibitory effect of miRNAs on target genes and upregulating the expression level of target genes, that is, functioning as competing endogenous RNA (ceRNA) [7]. circRNAs also bind to RNA binding proteins and may translate proteins to exert their functions $[8,9]$. circRNAs have critical regulatory effects in the occurrence and development of a variety of cancers, affecting cell cycle, apoptosis, metabolism, invasion, and metastasis [10]. circAGFG1 upregulates CCNE1 expression and promotes the proliferation, migration, and invasion of breast cancer cells [11]. circPPP1R12A-encoded protein circPPP1R12A-73aa promotes tumor growth and metastasis of colon cancer [12]. However, to date, little is known about the expression, functions, and regulatory mechanisms of circRNAs in LSCC.

Pre-B-cell leukemia homeobox transcription factor 3 (PBX3) is a member of the evolutionarily conserved three-amino acid-loop-extension (TALE) homeodomain transcription factor family. A recent study revealed that PBX3 is a critical regulatory protein of the epithelialmesenchymal transition (EMT) network in colorectal cancer [13]. Dysregulation of PBX3 expression has been observed in many cancer types, such as prostate, gastric, cervical, and liver cancer [14-17]. Nonetheless, the expression and function of PBX3 in LSCC are still unknown.

In this study, we performed RNA sequencing of 57 pairs LSCC tissues and matched adjacent normal mucosa (ANM) tissues and identified abnormally upregulated expression of circCORO1C in LSCC tissues. Furthermore, the expression of circCORO1C was strongly associated with the clinical features and prognosis of LSCC patients. We found that circCORO1C could bind to let-7c-5p and prevent it from decreasing the level of PBX3, which promoted EMT and stimulated the proliferation, migration, and invasion of LSCC cells in vitro and in vivo.

\section{Methods}

\section{LSCC patient tissue}

A total of 164 pairs of LSCC tissues and matched ANM tissues (taken $1-3 \mathrm{~cm}$ from the edge of cancer tissues) were obtained from patients undergoing surgery at the Department of Otolaryngology Head and Neck Surgery, The First Hospital of Shanxi Medical University, from January 2013 to January 2017. None of the patients received chemotherapy or radiotherapy before surgery. The tissue samples were diagnosed independently by two experienced clinical pathologists. The histological types of LSCC were determined according the World 
Health Organization (WHO) system, and TNM (Tumor, Node, Metastasis) stage was defined according to the criteria of the American Joint Committee on Cancer (AJCC, 8th edition). Fresh specimens were immediately frozen in liquid nitrogen. Among the 164 pairs of tissue samples, 57 paired LSCC (Additional file 1: Table S1) and ANM tissues were used for RNA sequencing, and 107 paired samples for qPCR analysis (Additional file 1: Table S2).

\section{Cell lines and cell culture}

Human LSCC cell line FD-LSC-1 (a gift from Professor Liang Zhou [18]) was cultured in BEGM ${ }^{\mathrm{mu}}$ Bronchial Epithelial Cell Growth Medium (Lonza, Walkersville, MD, USA) supplemented with 10\% FBS (Biological Industries, CT, USA). Human LSCC cell line TU-177 purchased from Bioleaf Biotech Corporation (Shanghai, China) was maintained in DMEM supplemented with 10\% FBS. Human HEK293T and MRC-5 cell lines were purchased from the China Center for Type Culture Collection (CCTCC). HEK293T cells were cultured in DMEM with $10 \%$ FBS. MRC-5 cells were cultured in MEM with $10 \%$ FBS. Human oral keratinocytes (HOK) purchased from ScienCell Research Laboratories (Carlsbad, CA) were cultured in DMEM with $10 \%$ FBS. All cells were cultured at $37{ }^{\circ} \mathrm{C}$ with $5 \% \mathrm{CO}_{2}$. Cell lines were tested for mycoplasma contamination using the TransDetect PCR Mycoplasma Detection Kit (TransGen Biotech, Beijing, China).

\section{RNA and genomic DNA (gDNA) extraction}

Total RNA was extracted from tissues or cells using Trizol reagent (Invitrogen, Waltham, MA) following the manufacturer's instructions. The nuclear and cytoplasmic fractions were extracted using a PARIS kit (ThermoFisher Scientific, Waltham, MA). gDNA was extracted using a genomic DNA isolation kit (TIANGEN Biotech (Beijing) Co., Ltd., Beijing, China).

\section{RNA sequencing analysis}

The RNA integrity of 57 pairs of LSCC/matched ANM tissues was examined with a Bioanalyzer 2100 (Agilent, Santa Clara, CA). High-quality RNA (RIN > 7) samples were subjected to library construction, and then each library was sequenced on an Illumina HiSeq 4000 (circRNA and mRNA) and Illumina HiSeq 2000 (miRNA) following the standard procedures by Novogene (Beijing, China). RNA sequencing data were deposited at GEO and are accessible via accession numbers GSE127165 and GSE133632. Differentially expressed circRNAs, miRNAs, and mRNAs were screened as reported [19] (Additional file 1: Table S3-5).
RT-PCR and quantitative real-time PCR (qPCR)

For PCR of mRNA and circRNA, RNA was reversetranscribed using a HiScript II 1st Strand cDNA Synthesis Kit (Vazyme, Nanjing, China). For qPCR of miRNA, cDNA was synthesized using an All-in-One ${ }^{\mathrm{Tw}}$ miRNA First-Strand cDNA Synthesis Kit (GeneCopoeia, Rockville, MD). qPCR was performed using ChamQ SYBR qPCR Master Mix (Vazyme, Nanjing, China) on an ABI Stepone Plus system. The relative expression levels were calculated using the $2^{(-\triangle \Delta C T)}$ method. The circRNA and mRNA levels were normalized by $18 \mathrm{~s}$ rRNA. The miRNA level was normalized against $U 6$ small nuclear RNA. Primer sequences are listed in Additional file 1: Table S6.

\section{RNase R treatment}

Total RNA $(2 \mu \mathrm{g})$ was incubated for $10 \mathrm{~min}$ at $37^{\circ} \mathrm{C}$ with or without $3 \mathrm{U} / \mu \mathrm{g}$ RNase R (Geneseed Biotech Co., Ltd., Guangzhou, China), followed by RNA purification using a RNeasy MinElute Cleanup kit (Qiagen, Hilden, Germany) and analyzed by RT-PCR.

\section{Agarose gel electrophoresis}

PCR products were separated by $2 \%$ agarose gel electrophoresis with TAE buffer using a $100 \mathrm{bp}$ DNA ladder (TransGen Biotech, Beijing, China). The bands were photographed under an Azure C600 imager (Azure Biosystems, Dublin, CA).

\section{Fluorescence in situ hybridization (FISH)}

Cy3-labeled circCORO1C probes (5'- AGAGCAATTG GTTCCTGCATATTTTTCTGGCAATCTCACATTTG

TTAACATC - 3') were synthesized by Sangon Biotech (Shanghai, China). FISH was performed using a FISH kit (RiboBio, Guangzhou, China) according to the manufacturer's instructions. Nuclei were stained with DAPI. Images were acquired on a Leica TCS SP8 confocal laser scanning microscope (Leica Microsystems Inc., Buffalo Grove, IL).

\section{Plasmid construction and cell transfection}

The PBX3 overexpression plasmid was generated by inserting $P B X 3$ CDS sequence into the $\mathrm{p} 3 \times$ FLAGCMV-10 vector (Sigma-Aldrich, St. Louis, MO). shRNA lentiviral plasmid targeting circCORO1C (psh-circCORO1C) was constructed by inserting annealed shRNA template DNA sequence into the PLKO.1 vector. For luciferase reporter plasmids, the sequences of cir$c$ CORO1C, wild type, and let-7c-5p binding site mutant $P B X 3$ 3' UTR were cloned into the psiCHECK-2 vector (Promega, Madison, WI). Cells were transfected using Lipofectamine 3000 (Invitrogen) according to the manufacturer's instructions. 
siRNAs, miRNA mimics, and inhibitor siRNAs targeting circCORO1C (si-circCORO1C \#1: 5' AGAUUGCCAGAAAAAUAUGCA-3'; si-circCORO1C \#2: 5' -UUGCCAGAAAAAUAUGCAGGA-3'), negative control siRNAs (si-NC), stable oligonucleotides (modified by cholesterol, 2'-OMe and phosphorothioate), let$7 c-5 p$ mimics, and NC were synthesized by GenePharma (Shanghai, China). miRNA inhibitor is small, chemically modified single-stranded RNA molecule that can competitively bind to and inhibit the function of specific endogenous mature miRNA. 2'-OMe-modified let-7c-5p inhibitor and $\mathrm{NC}$ inhibitor were synthesized by GenePharma.

\section{Generation of circCORO1C knockdown cells}

To generate FD-LSC-1 cells with stable knockdown of circCORO1C, lentiviruses were produced in HEK293T cells by cotransfection with psh-circCORO1C and packaging plasmids GAG and VSVG. Virus supernatant was harvested $48 \mathrm{~h}$ after transfection, mixed with polybrene $(8 \mu \mathrm{g} / \mathrm{ml})$, and added to FD-LSC- 1 cells. After $48 \mathrm{~h}$ incubation, $2 \mu \mathrm{g} / \mathrm{ml}$ puromycin (Santa Cruz Biotechnology, Dallas, TX) was added for 1 week to screen for stable cell clones.

\section{CCK8 assay}

After $24 \mathrm{~h}$ transfection, cells were digested and seeded into 96 -well plates $\left(3 \times 10^{3} /\right.$ well). At $0,24,48,72$, and $96 \mathrm{~h}$ after seeding, each well was replaced with $100 \mu \mathrm{L}$ fresh complete medium and $10 \mu \mathrm{L}$ TransDetect CCK (TransGen Biotech, Beijing, China) followed by incubation at $37^{\circ} \mathrm{C}$ with $5 \% \mathrm{CO}_{2}$ for $1 \mathrm{~h}$. The absorbance of the solution was measured at $450 \mathrm{~nm}$ using a Spectra Max i3x Multifunctional microplate detection system (Molecular Devices, San Jose, CA).

\section{5-Ethynyl-2'-deoxyuridine (EdU) staining}

Cells were incubated with DMEM medium containing $50 \mu \mathrm{M}$ EdU (RiboBio) at $37^{\circ} \mathrm{C}$ with $5 \% \mathrm{CO}_{2}$ for $2 \mathrm{~h}$. Cells were washed twice with PBS, fixed with $50 \mu \mathrm{L} 4 \%$ paraformaldehyde for $30 \mathrm{~min}$, neutralized with $50 \mu \mathrm{L} 2$ $\mathrm{mg} / \mathrm{mL}$ glycine solution and permeabilized by adding $100 \mu \mathrm{L} 0.5 \%$ Triton X-100. After washing with PBS, $100 \mu \mathrm{L} 1 \times$ Apollo dye was added to each well, then cells were incubated at room temperature for $30 \mathrm{~min}$. Next, $100 \mu \mathrm{L}$ Hoechst 33342 was added and incubated for another $30 \mathrm{~min}$. Images were captured and analyzed on an ImageXpress high-content screening system (Molecular Devices).

\section{Colony formation assay}

Transfected cells were seeded at a density of 600 cells/ well into a 35-mm dish and then cultured for 10 days. Cells were washed with PBS once and colonies were fixed with $4 \%$ paraformaldehyde for $20 \mathrm{~min}$ and stained with $0.1 \%$ crystal violet solution for $10 \mathrm{~min}$ at room temperature, followed by image capture.

\section{Transwell migration and invasion assays}

After $24 \mathrm{~h}$ transfection, cells were digested, washed twice with PBS and resuspended in serum-free DMEM. Transwell chambers for invasion assay were precoated with Matrigel (BD Biosciences, San Jose, CA). Serum-free DMEM $(200 \mu \mathrm{L})$ containing cells $\left(4 \times 10^{4}\right.$ cells/well for migration assay, $1 \times 10^{5}$ cells/well for invasion assay) was added to the upper chamber. Then $500 \mu \mathrm{L}$ DMEM medium supplemented with $20 \%$ FBS was added to the lower chamber. After $24 \mathrm{~h}$, cells in the upper chamber were removed with cotton swabs and the lower side of the chamber was gently washed twice with PBS, fixed with $4 \%$ paraformaldehyde for $20 \mathrm{~min}$, and stained with $0.1 \%$ crystal violet for $10 \mathrm{~min}$, and then images were captured by microscope.

\section{Apoptosis analysis}

Apoptosis was determined using a Dead Cell Apoptosis kit (ThermoFisher Scientific). Briefly, cells were digested with EDTA-free trypsin and washed with ice-cold PBS, followed by a 15-min incubation with Alexa Fluor 488 annexin $\mathrm{V}$ and PI, then cells were analyzed by a NovoCyte flow cytometer (ACEA Biosciences, Hangzhou, China).

\section{Prediction of RNA interaction}

Target gene prediction of $l e t-7 c-5 p$ was performed using the ENCORI online program with strict stringency (http://starbase.sysu.edu.cn/index.php). The interaction between circCORO1C and miRNA was predicted by seedVicious v1.0 and RegRNA 2.0 (https://seedvicious. essex.ac.uk/predict.html, http://regrna2.mbc.nctu.edu.tw/ index.html).

\section{TCGA data analysis}

Transcriptome sequencing data and clinical features of head and neck squamous cell carcinoma (HNSCC) were downloaded from The Cancer Genome Atlas (TCGA) HNSCC cohort (https://portal.gdc.cancer.gov/projects/ TCGA-HNSC), followed by expression analysis of $P B X 3$ and $l e t-7 c-5 p$ with normalized FPKM and RPM values.

\section{RNA immunoprecipitation (RIP)}

RIP experiments were performed with a Magna RIP RNA-Binding Protein Immunoprecipitation Kit (Millipore, Billerica, MA) according to the manufacturer's instructions. Briefly, $1 \times 10^{7}$ cells were collected and resuspended in $300 \mu \mathrm{L}$ RIPA lysis buffer containing protease inhibitor cocktail and RNase inhibitors. The cell lysates $(200 \mu \mathrm{L})$ were incubated with $5 \mu \mathrm{g}$ AGO2 
antibody (\#2897; CST, Danvers, MA) or rabbit IgG and protein $\mathrm{A} / \mathrm{G}$ magnetic beads at $4{ }^{\circ} \mathrm{C}$ overnight with rotation. Immunoprecipitated RNA was purified using a RNeasy MinElute Cleanup kit (Qiagen). The enrichment of $\operatorname{circCORO1C}$ was evaluated by qPCR.

\section{Luciferase reporter assay}

HEK293T cells were cotransfected with luciferase reporter plasmid and let-7c-5p mimics or $\mathrm{NC}$ mimics for $48 \mathrm{~h}$. The luciferase activity was measured using a dual luciferase reporter assay system (Promega) on a Spectra Max i3x Multifunctional microplate detection system (Molecular Devices). The luciferase values were normalized and then the relative luciferase activity was calculated.

\section{Western blotting}

Total protein was extracted with RIPA buffer containing protease inhibitor cocktail (ThermoFisher Scientific). The protein concentration was determined using a Coomassie (Bradford) Protein Assay Kit (ThermoFisher Scientific). Equal amounts of total protein $(30 \mu \mathrm{g})$ were separated by $12 \%$ SDS-PAGE and transferred onto PVDF membranes (Millipore, Billerica, MA), followed by blocking with $5 \%$ skim milk. The membranes were incubated with antibodies against PBX3 (\#12571-1-AP; Proteintech, Wuhan, China), E-cadherin (\#3195S; CST, Danvers, MA), N-cadherin (\#13116S; CST), Vimentin (\#5741S; CST), Slug (\#sc-166476; Santa Cruz Biotechnology), or GAPDH (\#HC301-02; TransGen Biotech) overnight at $4{ }^{\circ} \mathrm{C}$. Then membranes were washed three times with TBST followed by secondary antibody incubation for $2 \mathrm{~h}$ at room temperature. Bands were detected by a chemiluminescence imaging system (SageCreation Science, Beijing, China) with Western Bright ECL HRP substrate (Advansta Inc., San Jose, CA).

\section{Xenograft tumorigenesis}

SPF-grade male BALB/C nude mice (6-8 weeks) were purchased from Beijing Vital River Laboratory Animal Technology Co., Ltd. (Beijing, China) and housed under SPF conditions (TECNIPLAST S.p.A., Italy). A total of $2 \times 10^{6}$ FD-LSC-1 cells were suspended in $200 \mu \mathrm{L}$ serum-free DMEM and subcutaneously injected into the right flank of each mouse. The volumes of tumors were measured from 7 days after injection. Tumor volume was calculated as follows: V (volume) $=($ length $\times$ width $\left.^{2}\right) / 2$. After 25 days, the mice were killed and the tumors were dissected, weighed, and processed for histological analysis.

\section{Immunohistochemical (IHC) staining}

IHC staining was performed as previously described [3]. In brief, tissues were fixed in $4 \%(\mathrm{v} / \mathrm{v})$ formaldehyde in
PBS, embedded in paraffin, and cut into $3-\mu \mathrm{m}$ sections. Sections underwent dewaxing, re-hydration, antigen retrieval, and blocking, and then were incubated with antibodies against PBX3, Ki67, E-cadherin, N-cadherin, and Vimentin overnight at $4{ }^{\circ} \mathrm{C}$ in a moist chamber, and washed three times with PBST. Sections were incubated with HRP-conjugated secondary antibody (CST) for 15 $\mathrm{min}$ at room temperature, washed three times with PBST, and then stained with DAB and hematoxylin. Next, sections were dehydrated and mounted with coverslips.

\section{Statistical analysis}

Statistical analysis was performed using GraphPad Prism 7.0 software (La Jolla, USA). Comparisons between two groups were performed using the two-tailed Student's $t$ test. Correlations were analyzed by Pearson's correlation. Kaplan-Meier survival curve and log-rank test were employed to depict the overall survival probability of LSCC patients with different expression levels of cir$c$ CORO1C. Results are presented as mean \pm standard deviation (SD). $P$ values of $<0.05$ were considered statistically significant.

\section{Results \\ circCORO1C is frequently upregulated in LSCC and is associated with malignant progression and poor prognosis}

We performed RNA sequencing in 57 pairs of LSCC and matched ANM tissues. Differential expression screening showed that 410 circRNAs were upregulated in LSCC tissues (Additional file 1: Table S3), in which 18 circRNAs were detected in all sequenced tissues (Fig. 1a). Expression of the 18 circRNAs was verified by RT-PCR and Sanger sequencing, and 12 circRNAs were validated successfully (Additional file 2: Figure S1a and b). Next, we screened circRNAs that affect LSCC proliferation by siRNA-mediated knockdown and high-content screening. We found that knockdown of circRNA hg19_circ 0008714 significantly inhibited LSCC cell proliferation (Additional file 2: Figure S1c). Hence, we focused on this circRNA in this study. Sequence analysis revealed that hg19_circ_0008714 was formed by back-splicing of exons 7 and 8 of the Coronin-like actin-binding protein $1 \mathrm{C}$ gene $(C O R O 1 C)$ and was therefore named circCORO1C (Fig. 1b). RT-PCR and Sanger sequencing were performed to verify the expression and head-to-tail splicing of circCORO1C in LSCC (Fig. 1b). Moreover, we compared the expression levels of circCORO1C in LSCC cells and normal control cell lines by qPCR. The results showed that the expression levels of circCORO1C in LSCC cell lines FD-LSC-1 and TU-177 were significantly higher than those in normal control cell lines HEK293T, HOK, and MRC-5 (Fig. 1c). 
A

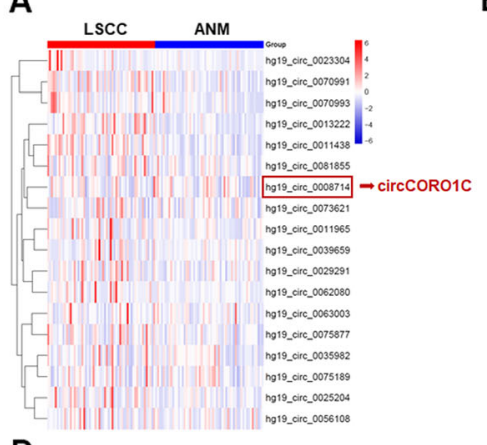

D
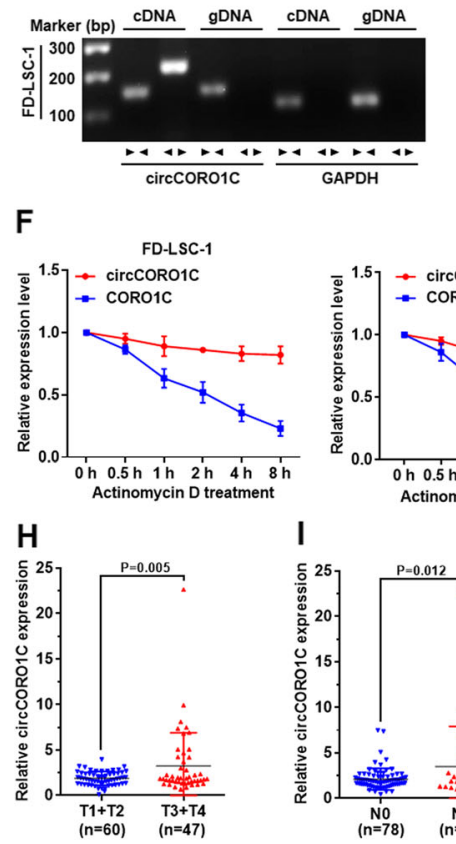

L

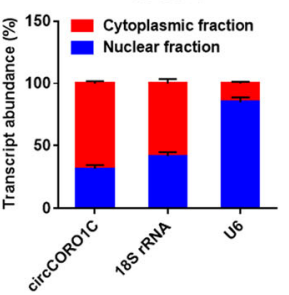

B



Marker (bp) cDNA gDNA cDNA gDNA



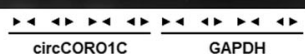

\section{G}

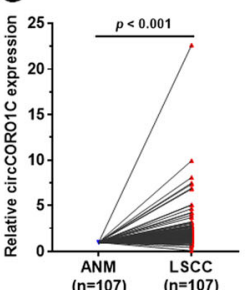

$\underset{\text { TU-177 }}{1.5} \rightarrow$ circCORO1C


J $\mathbf{K}$



$\mathrm{K}$

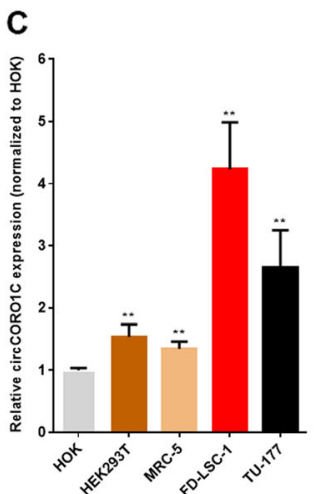

E
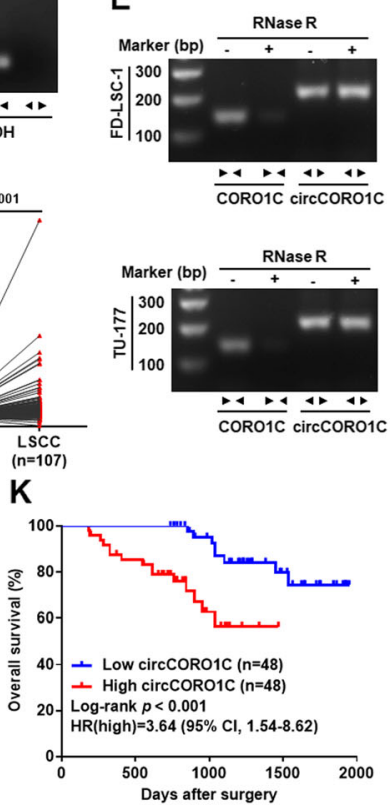

M
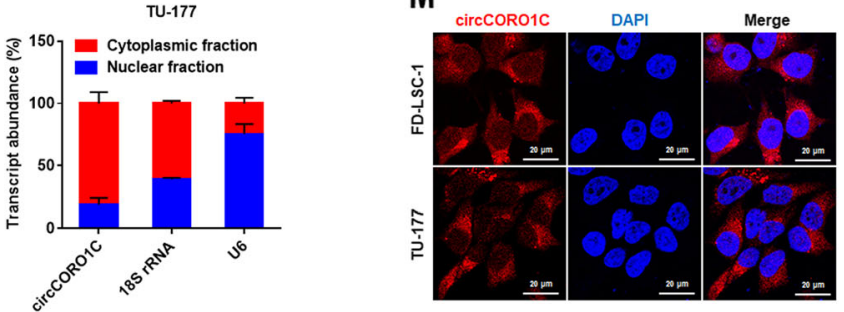

Fig. 1 circCORO1C is upregulated in LSCC tissues and is associated with poor prognosis. a RNA sequencing of 57 pairs of LSCC and matched adjacent normal mucosal (ANM) tissues to screen differentially expressed circRNAs. Heatmap showing circRNAs expressed in all tissues and those upregulated in LSCC tissues. b Schematic illustration showed the circularization of CORO1C exons 7 and 8 to form circCORO1C. The back-splicing junction of circCORO1C was verified by RT-PCR and Sanger sequencing. c circCORO1C expression levels in human LSCC cell lines (FD-LSC-1, TU-177) and normal cell lines (HOK, HEK293T, MRC-5) were determined by qPCR. d circCORO1C expression in FD-LSC-1 and TU-177 cells verified by RT-PCR. Agarose gel electrophoresis showed that divergent primers amplified circCORO1C in CDNA but not genomic DNA (gDNA). GAPDH served as a negative control. e Validation of circCORO1C stability by RNase R treatment and RT-PCR analysis. $\mathbf{f}$ qPCR analysis of the abundance of circCORO1C and linear CORO1C in FD-LSC-1 and TU-177 cells treated with actinomycin D at the indicated times. $\mathbf{g}$ Expression levels of circCORO1C in an additional 107 paired LSCC tissues were determined by qPCR. $\mathbf{h}$-j Correlation analysis between circCORO1C expression levels and clinicopathological parameters of LSCC. Expression level of circCORO1C was significantly associated with T stage (h), N stage (i), and clinical stage (j). k Kaplan-Meier analysis of the correlation between circCORO1C expression and overall survival of 96 patients with LSCC. I circCORO1C abundance in nuclear and cytoplasmic fractions of FD-LSC1 and TU-177 cells was evaluated by qPCR. 18S RNA acted as a positive control of RNA distributed in the cytoplasm, and U6 RNA acted as a positive control of RNA distributed in the nucleus. $\mathbf{m}$ Localization of circCORO1C in FD-LSC-1 and TU-177 cells was detected by FISH. Nuclei were stained with DAPI (blue) and circCORO1C probes were labeled with Cy3 (red). The error bars (c, $\mathbf{f}$ and $\mathbf{I}$ ) represent SD of three independent experiments. ${ }^{*} P<0.001$ 
Head-to-tail splicing sequences may be produced by mechanisms other than the formation of circRNA, such as trans-splicing and genomic rearrangements [20]. To rule out the possibility of the latter two, convergent primers for linear and special divergent primers for circular RNA were designed. The convergent primers could be amplified when the template contained CORO1C linear mRNA or genomic DNA (gDNA), while the divergent primers could only be specifically amplified in the presence of circCORO1C. cDNA and gDNA from FDLSC-1 and TU-177 cells, respectively, were used as PCR templates. Nucleic acid electrophoresis results indicated that the divergent primers could amplify circCORO1C only in CDNA, but no products were detected in the gDNA (Fig. 1d). High stability is a crucial feature of cir-


was used to pretreat the RNA; the results demonstrated that linear CORO1C mRNA was significantly reduced after RNase $\mathrm{R}$ treatment, while $\operatorname{circCORO1C}$ was resistant to RNase R (Fig. 1e). Moreover, we further compared the half-life of the circular form and linear CORO1C through treatment with RNA transcription inhibitor actinomycin D and qPCR assay, and found that circCORO1C had a significantly longer half-life than the linear CORO1C (Fig. 1f). These data confirmed essential features of $\operatorname{circCORO1C\text {.}}$

To investigate the correlation between circCORO1C levels and LSCC, we detected the expression of circCORO1C in 107 pairs of LSCC and ANM tissues by qPCR. The relative abundance of circCORO1C in LSCC tissues was significantly higher than that in ANM tissues (Fig. 1g). Moreover, the expression level of circCORO1C was significantly correlated with $\mathrm{T}$ stage, $\mathrm{N}$ stage, and clinical stage. Patients in the advanced stage and those with cervical lymph node metastasis had high expression levels of circCORO1C (Fig. 1h-j). Importantly, KaplanMeier analysis revealed that LSCC patients with high circCORO1C levels had poor overall survival (Fig. 1k).

Next, we investigated the location of circCORO1C in cells by nuclear and cytoplasmic RNA extraction and qPCR. The results showed that circCORO1C was mainly localized to the cytoplasm (Fig. 11). FISH further confirmed that circCORO1C was mainly localized to the cytoplasm (Fig. 1m). These results indicated that circCORO1C upregulation is common in LSCC and may have important functions in the progression of LSCC.

\section{circCORO1C promotes the proliferation, migration, and invasion of LSCC cells}

To investigate the functions of $\operatorname{circCORO1C}$ in LSCC cells, we designed and synthesized two siRNAs that specifically targeted the back-splicing region of circCORO1C. After LSCC cell lines FD-LSC-1 and TU-177 were transfected with siRNA, qPCR was performed to evaluate the knockdown efficiency. The results showed that both siRNAs could significantly reduce circCORO1C, while the level of linear CORO1C was not significantly changed, and siRNA \#1 had the highest knockdown efficiency (Fig. 2a). We also tested cell viability by CCK- 8 assay, and found that knockdown of circCORO1C significantly inhibited the viability of LSCC cells (Fig. 2b). EdU staining experiments confirmed that knockdown of circCORO1C inhibited the proliferation of LSCC cells (Fig. 2c). Colony formation experiments showed that knockdown of circCORO1C significantly inhibited the colony formation of FD-LSC-1 and TU-177 cells (Fig. 2d).

Furthermore, we investigated the effects of circCORO1C on the migration and invasion of LSCC cells by Transwell assays. Knockdown of circCORO1C significantly decreased cell migration and invasion (Fig. $2 \mathrm{e}$ and $\mathrm{f}$ ). We further investigated the effect of circCORO1C on apoptosis, and found that knockdown of circCORO1C promoted apoptosis in LSCC cells (Fig. 2g). Taken together, these findings suggested that circCORO1C has an oncogenic role in LSCC, and si-circCORO1C \#1 had the strongest effect on the cell functions, which was consistent with the knockdown efficiency. Thus, in subsequent studies, we performed experiments using si-circCORO1C \#1.

\section{circCORO1C acts as a miRNA sponge of let-7c-5p in LSCC cells}

Studies have showed that circRNAs can function as miRNA sponges to competitively bind to miRNA, thus abrogating the inhibitory effect of miRNA on downstream target genes. Since $\operatorname{circCORO1C}$ is distributed in the cytoplasm, we studied whether it could function as a miRNA sponge in LSCC cells. We used the online tools RegRNA and seedVicious to predict the circCORO1Cbinding miRNA (Additional file 1: Table S7), and intersected the data with miRNAs that were found to be downregulated in RNA sequencing of our 57 pairs of LSCC and ANM tissues. Notably, let-7c-5p was the only one common miRNA in these three datasets (Fig. 3a).

As one of the critical components of RNA-induced silencing complex (RISC), Argonaute 2 (AGO2) is the major protein that mediates the interaction between circRNA and target miRNAs [21]. To demonstrate that cir$c C O R O 1 C$ functions as a miRNA sponge, we performed RIP assay using AGO2 antibody in FD-LSC-1 and HOK cells. The results showed that circCORO1C was pulled down with AGO2 and was less enriched in HOK cells compared with FD-LSC-1 cells, which consistent with the differential circCORO1C levels of these two cell lines (Fig. 3b). In addition, the results of RIP in let-7c-5ptransfected FD-LSC-1 cells indicated that circCORO1C was specifically enriched by $\mathrm{AGO} 2$ antibody (Additional 


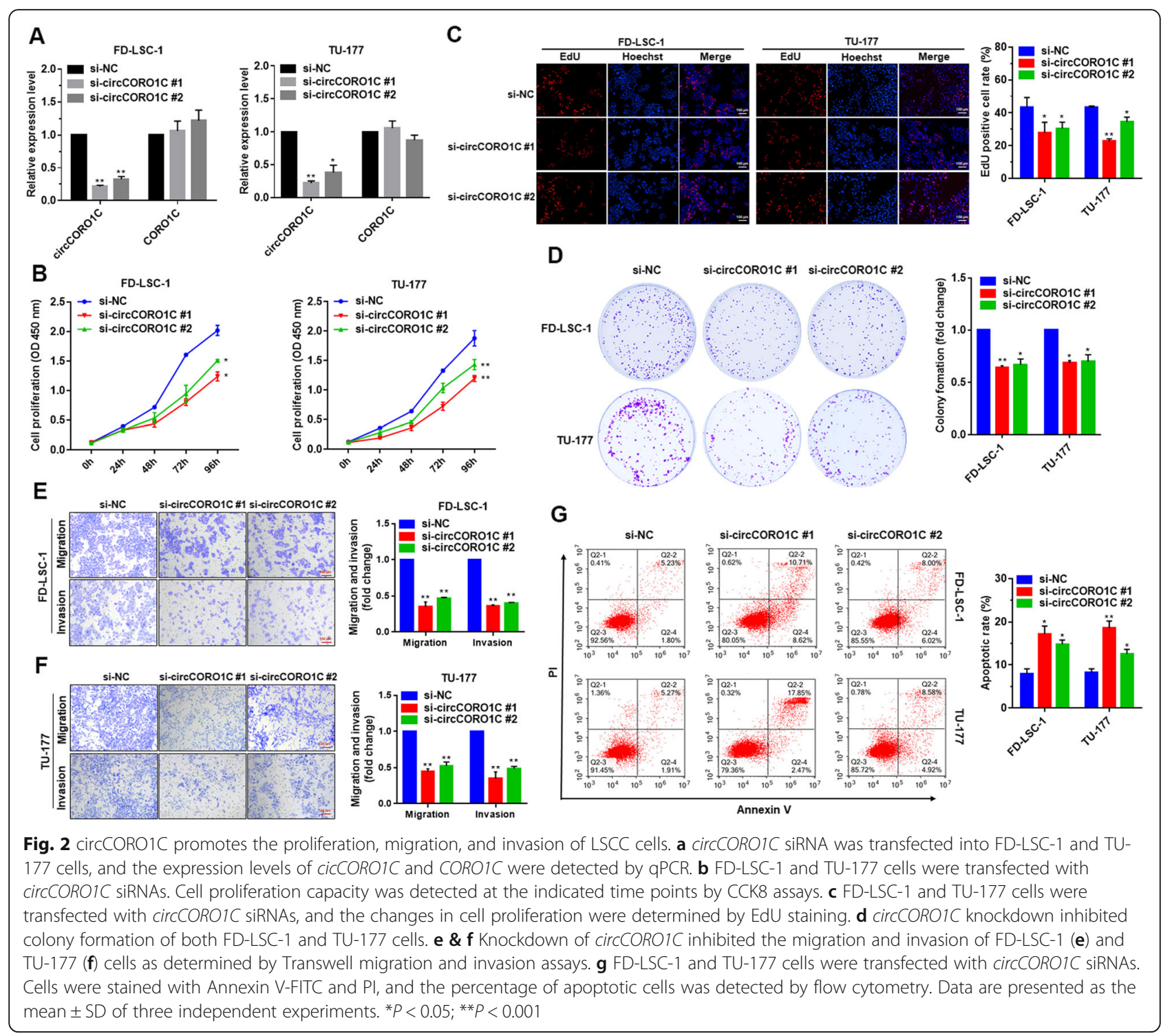

file 2: Figure S2). Moreover, we constructed luciferase reporter vectors for wild-type $\operatorname{circCORO1C}$ and let-7c-5p binding site mutant circCORO1C and co-transfected HEK293T cells with let-7c-5p mimics or NC mimics. The results showed that the luciferase activity in the wild-type group co-transfected with let-7c-5p mimics was significantly reduced, while the luciferase activity in the binding site mutant group was not significantly changed (Fig. 3c). Furthermore, the expression correlation between circCORO1C and let-7c-5p was analyzed by qPCR in 20 pairs of LSCC tissues, revealing that let$7 c-5 p$ levels were negatively correlated with circCORO1C levels in LSCC tissues (Fig. 3d). In addition, we found that expression of let-7c-5p in FD-LSC-1 and TU-177 cells was increased significantly after circCORO1C knockdown (Fig. 3e). Collectively, these results indicated that $\operatorname{circCORO1C}$ functions as a miRNA sponge to directly interact with let-7c-5p in LSCC cells.

\section{let-7c-5p is downregulated in LSCC tissues and inhibits the malignant phenotype of LSCC cells}

RNA sequencing data indicated that let-7c-5p expression levels in LSCC tissues were significantly lower than those in ANM tissues (Fig. 4a). Analysis of TCGA data confirmed that let-7c-5p expression was downregulated in HNSCC and LSCC (Fig. 4b and c), indicating that let$7 c-5 p$ may have important roles in LSCC. Therefore, we investigated the functions of let-7c-5p in LSCC cells. FD-LSC-1 and TU-177 cells were transfected with let$7 c-5 p$ mimics or negative control mimics (NC mimics), then the transfection efficiency was verified by qPCR, which revealed that let-7c-5p expression was elevated 



E
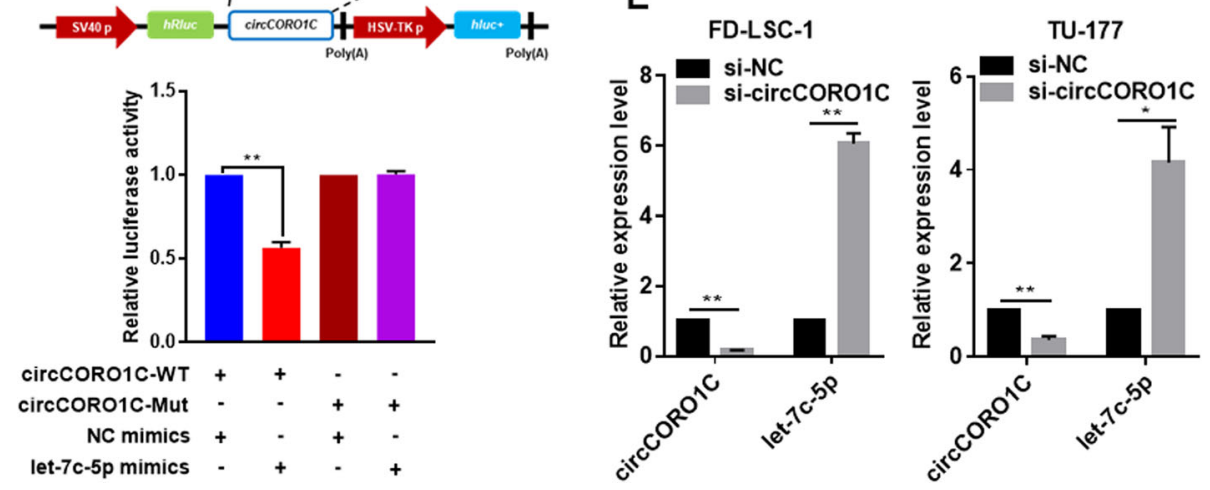

Fig. 3 circCORO1C acted as a sponge for miRNA let-7C-5p in LSCC cells. a Combined analysis of bioinformatics prediction and LSCC tissue RNA sequencing data to screen for circCORO1C-binding miRNAs. $\mathbf{b}$ RIP assays were performed using AGO2 antibody in FD-LSC-1 and HOK cells, then the enrichment of circCORO1C was detected by qPCR. c HEK293T cells were co-transfected with let-7c-5p mimics and wild-type or mutant circCORO1C luciferase reporter vector, and luciferase reporter activity was detected. $\mathbf{d}$ Correlation analysis of circCORO1C and let-7c-5p RNA levels in 20 pairs of LSCC tissues. Expression of circCORO1C and let-7c-5p in 20 cases of LSCC and matched ANM tissues was determined by qPCR, and the relative expression of cirCCORO1C and let-7C-5p was normalized to ANM. e Expression levels of circCORO1C and let-7C-5p in FD-LSC-1 and TU177 cells transfected with circCORO1C siRNAs were evaluated by qPCR. Data are presented as the means \pm SD of three independent experiments. ${ }^{*} P<0.05 ;{ }^{* *} P<0.001$

(Fig. 4d). CCK8 assay and EdU staining indicated that overexpression of let-7c-5p inhibited LSCC cell proliferation (Fig. 4e and f). Colony formation experiments found that the colony formation ability of LSCC cells overexpressing let-7c-5p was significantly decreased (Fig. 4g), while Transwell assay showed that the migration and invasion of LSCC cells were significantly attenuated after overexpression of let-7c-5p (Fig. $4 \mathrm{~h}$ and i). Apoptosis assay showed that let-7c-5p overexpression promoted the apoptosis of LSCC cells (Fig. 4j). Overall, these data demonstrated that let- $7 c-5 p$ inhibits the proliferation, migration, and invasion of LSCC cells and promotes their apoptosis.

\section{let-7c-5p reversed the tumor-promoting effects of circCORO1C in LSCC cells}

To identify whether circCORO1C promoted LSCC cell proliferation, migration, and invasion by interacting with let- $7 c-5 p$, we conducted a rescue experiment. FD-LSC-1 and TU-177 cells were cotransfected with si-cir$c$ CORO1C and let-7c-5p inhibitor (Fig. 5a), and then CCK-8, EdU staining, and colony formation assays were conducted. The results showed that when let-7c-5p function was inhibited with miRNA inhibitor, the proliferation and colony formation ability of LSCC cells was significantly enhanced (Fig. 5b-d). Notably, transfection with let-7c-5p inhibitor could reverse the decreased cell 


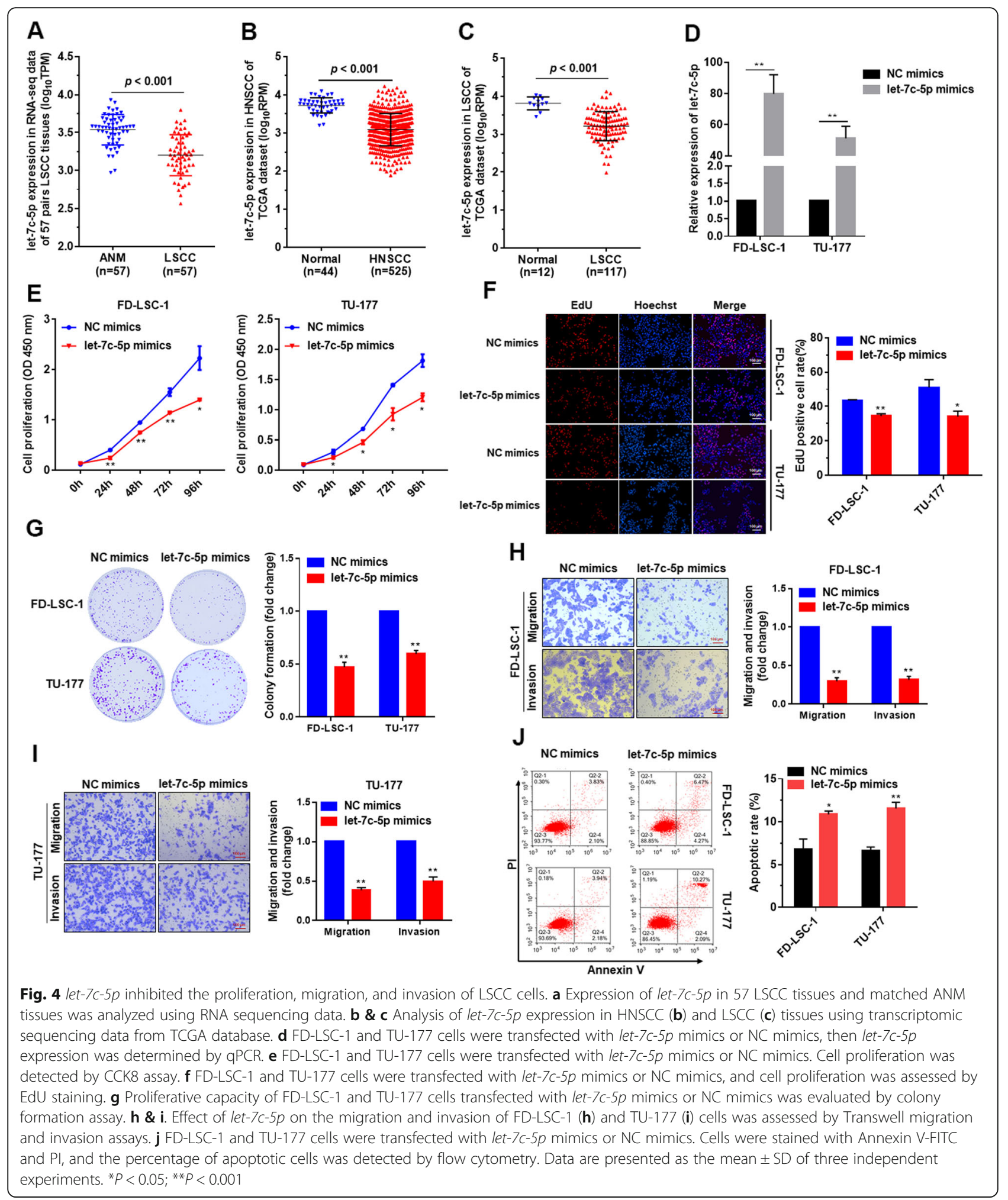

viability caused by si-circCORO1C (Fig. 5b-d). Transwell assays showed that let-7c-5p inhibition reversed the reduction in the migration and invasion of FD-LSC-1 and TU-177 cells caused by circCORO1C knockdown (Fig. 5e). Compared with cells transfected with si-circCORO1C alone, co-transfection of LSCC cells with sicircCORO1C and let-7c-5p inhibitor significantly reduced apoptosis (Fig. 5f). Taken together, these results 


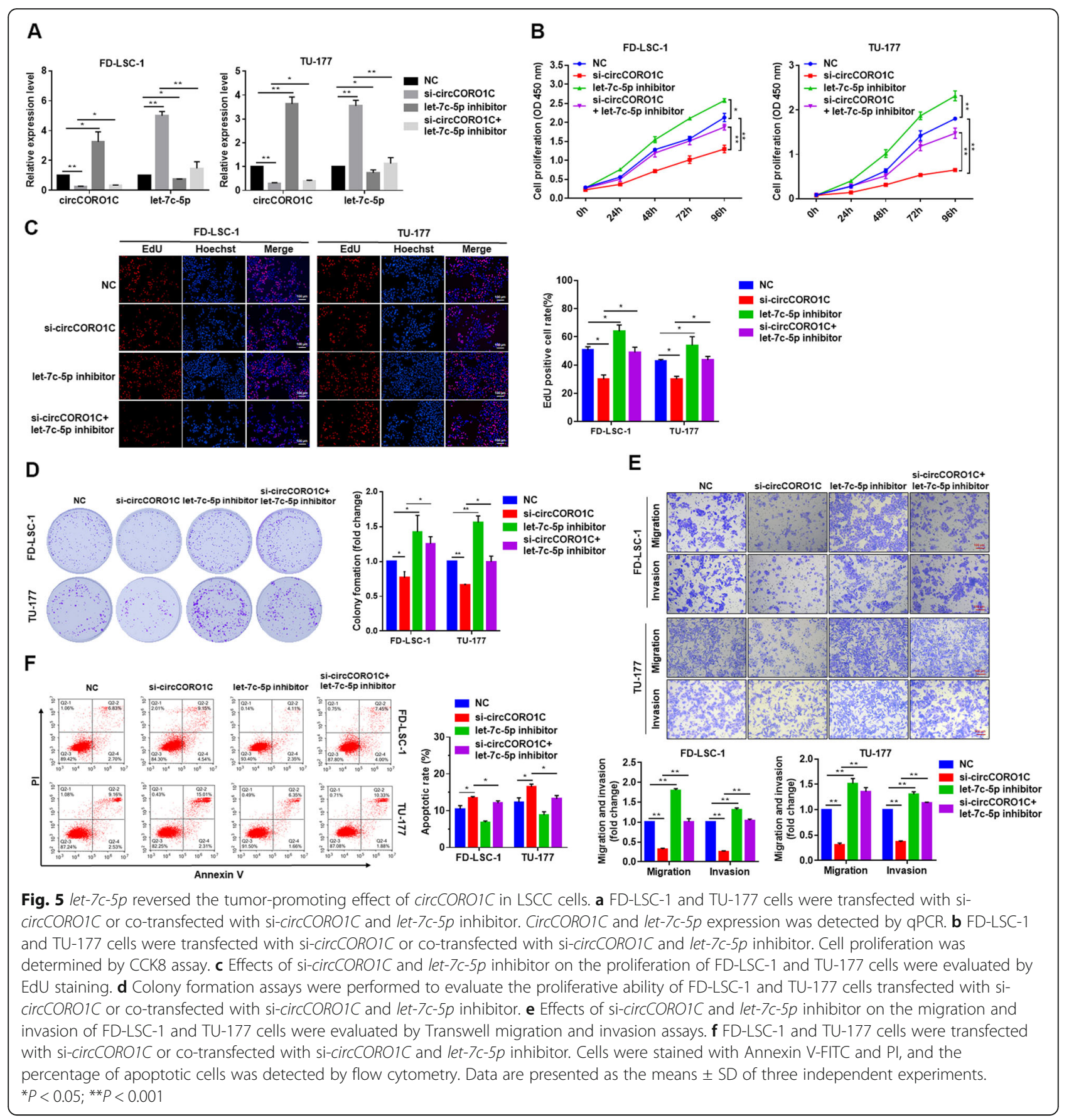

indicated that circCORO1C promoted the malignant progression of LSCC cells mainly by abolishing the antitumor effect of let-7c-5p.

$P B X 3$ is a direct target of let-7c-5p and functions as driver gene in LSCC

According to ceRNA theory, $\operatorname{circCORO1C}$ is positively correlated with the expression of downstream target genes, while the target gene is negatively correlated with let-7c-5p expression. We predicted the possible let-7c-5p target genes by miRanda, PicTar, PITA, and TargetScan, and 257 genes intersected by these four programs were obtained (Fig. 6a; Additional file 1: Table S8). Then we intersected these 257 genes with the mRNAs that were found to be upregulated in LSCC tissues upon RNA sequencing, and 51 intersected genes were obtained (Fig. 6b; Additional file 1: Table S9). Next, we analyzed the expression correlation of $\operatorname{circCORO1C}$ and let-7c-5p with the 51 genes using RNA sequencing data of 57 pairs of LSCC samples. Pearson correlation analysis indicated 




that $\operatorname{circCORO1C}$ was positively correlated with $P B X 3$, while let-7c-5p was negatively correlated with $P B X 3$ in LSCC and ANM tissues (Fig. 6c and d). RNA sequencing data showed that $P B X 3$ was upregulated in $73.7 \%(42 / 57)$ of LSCC tissues (Fig. 6ec and d). Moreover, analysis of the transcriptomic data of TCGA database found that $P B X 3$ was upregulated in both HNSCC and LSCC (Fig. 6f). In addition, overexpression of let-7c-5p significantly decreased the expression of PBX3 mRNA and protein (Fig. 6g), while downregulation of let-7c-5p remarkably increased it in FDLSC-1 and TU-177 cells (Fig. 6h). 
To demonstrate that let-7c-5p interacts directly with the 3' UTR of $P B X 3$, we constructed wild-type (WT) $P B X 3$ 3' UTR and let-7c-5p binding-site mutant (Mut) luciferase reporter plasmids. The wild-type and mutant reporter vectors were co-transfected with let$7 c-5 p$ mimics in cells. Luciferase reporter assays showed that let-7c-5p mimics significantly decrease the luciferase activity of WT, while the luciferase activity of the Mut group was not significantly changed (Fig. 6i), indicating that let-7c-5p suppresses PBX3 expression by directly binding to the $3^{\prime}$ UTR of $P B X 3$ mRNA.

Subsequently, we investigated the functions of PBX3 in LSCC cells. Overexpression of PBX3 promoted the proliferation, migration, and invasion of LSCC cells (Fig. $6 \mathbf{j}-1)$. Notably, we observed that overexpression of PBX3 counteracted the inhibitory effects of let-7c-5p on LSCC cell proliferation, migration, and invasion (Fig. 6j-l). Collectively, these findings suggested that $P B X 3$ is a driver gene and a direct target of let-7c-5p in LSCC.

\section{circCORO1C facilitates the malignant progression of LSCC cells by targeting PBX3}

To investigate whether circCORO1C promoted the malignant progression of LSCC cells by regulating the downstream target gene $P B X 3$, we simultaneously transfected $P B X 3$ overexpression plasmid and si-circCORO1C into FD-LSC-1 and TU-177 cells (Fig. 7a) and detected changes in the cell phenotypes. CCK- 8 assay and EdU staining were performed, and the results showed that overexpression of $P B X 3$ could inhibit the decrease in cell proliferation caused by circCORO1C knockdown (Fig. 7b and c). Consistently, overexpression of $P B X 3$ rescued the decreased colony formation ability by circCORO1C knockdown (Fig. 7d). Furthermore, Transwell assay showed that overexpression of $P B X 3$ could reverse the decline in cell migration and invasion ability caused by circCORO1C knockdown (Fig. 7e). We also detected protein changes in EMT marker genes by western blotting. circCORO1C knockdown enhanced the expression of E-cadherin while inhibiting the expression of $\mathrm{N}$ cadherin, Vimentin, and Slug (Fig. 7f), and overexpression of $P B X 3$ could reverse the regulatory effects of circCORO1C on these EMT markers. Compared with the si-circCORO1C group, the expression of E-cadherin was reduced, and the expression of $\mathrm{N}$-cadherin, Vimentin, and Slug were increased in the group cotransfected with si-circCORO1C and $P B X 3$ overexpression plasmid (Fig. 7f). These findings indicated that $\operatorname{circCORO1C}$ promoted the proliferation, migration, and invasion phenotype of LSCC cells by specifically upregulating the expression of the target gene $P B X 3$ and affecting the EMT process at the same time.
circCORO1C enhances the growth of xenograft tumors of LSCC cells in vivo

To investigate the regulatory effect of $\operatorname{circCORO1C}$ on LSCC under in vivo conditions, we constructed a shRNA lentiviral plasmid targeting circCORO1C and screened FD-LSC-1 cells following stable knockdown of circCORO1C (sh-circCORO1C). Next, we constructed xenograft tumor models of nude mice by subcutaneously injecting stably transfected FD-LSC-1 cells. The xenograft tumors formed by circCORO1C-deficient LSCC cells had a significantly smaller volume than those of the control group (sh-NC) (Fig. 8a), and the tumor weight was also significantly lower than the sh-NC group (Fig. $8 \mathrm{~b})$. The total RNA of xenograft tumors was extracted, and $\mathrm{qPCR}$ was used to detect the expression of cir$c C O R O 1 C$, let- $7 c-5 p$, and $P B X 3$. The results confirmed decreased circCORO1C and $P B X 3$ expression, while let$7 c-5 p$ was increased in tumors with circCORO1C knockdown (Fig. 8c). Furthermore, hematoxylin and eosin (H\&E) staining showed that knockdown of circCORO1C remarkably reduced the number of lesions (Fig. 8d). IHC staining demonstrated that the expression of PBX3 and proliferation marker Ki67 was decreased in sh-circCORO1C xenograft tumors (Fig. 8e). In addition, the changes in EMT marker E-cadherin, N-cadherin, Vimentin, and Slug expression were determined by IHC. The results revealed that knockdown of circCORO1C attenuated the mesenchymal phenotype (Fig. 8e). These data confirmed that circCORO1C promoted the malignant progression of LSCC in vivo.

\section{Discussion}

Studies have shown that circRNA has important regulatory effects in a variety of biological processes, especially in the occurrence, development, and metastasis of various malignant tumors [22-24]. circRNA expression profiling revealed a series of differentially expressed circRNAs in LSCC tissues [25, 26], suggesting that circRNA may have important roles in the occurrence and progression of LSCC. In this study, we performed largescale RNA sequencing of LSCC and matched ANM tissues, and established the circRNA, miRNA, and mRNA expression profiles of LSCC tissues. We identified and verified that $\operatorname{circCORO1C}$ was highly expressed in LSCC tissues and cells, and its expression levels were correlated with clinicopathological parameters and LSCC patient survival. Loss-of-function experiments demonstrated that $\operatorname{circCORO1C}$ promoted the proliferation, migration, and invasion of LSCC cells and inhibited their apoptosis. Mechanistic studies showed that $\operatorname{circCORO1C}$ bound to let-7c-5p and attenuated the inhibition of let$7 c-5 p$ on the target gene $P B X 3$, leading to PBX3 accumulation and enhancing the proliferation, migration, and invasion of LSCC cells. 







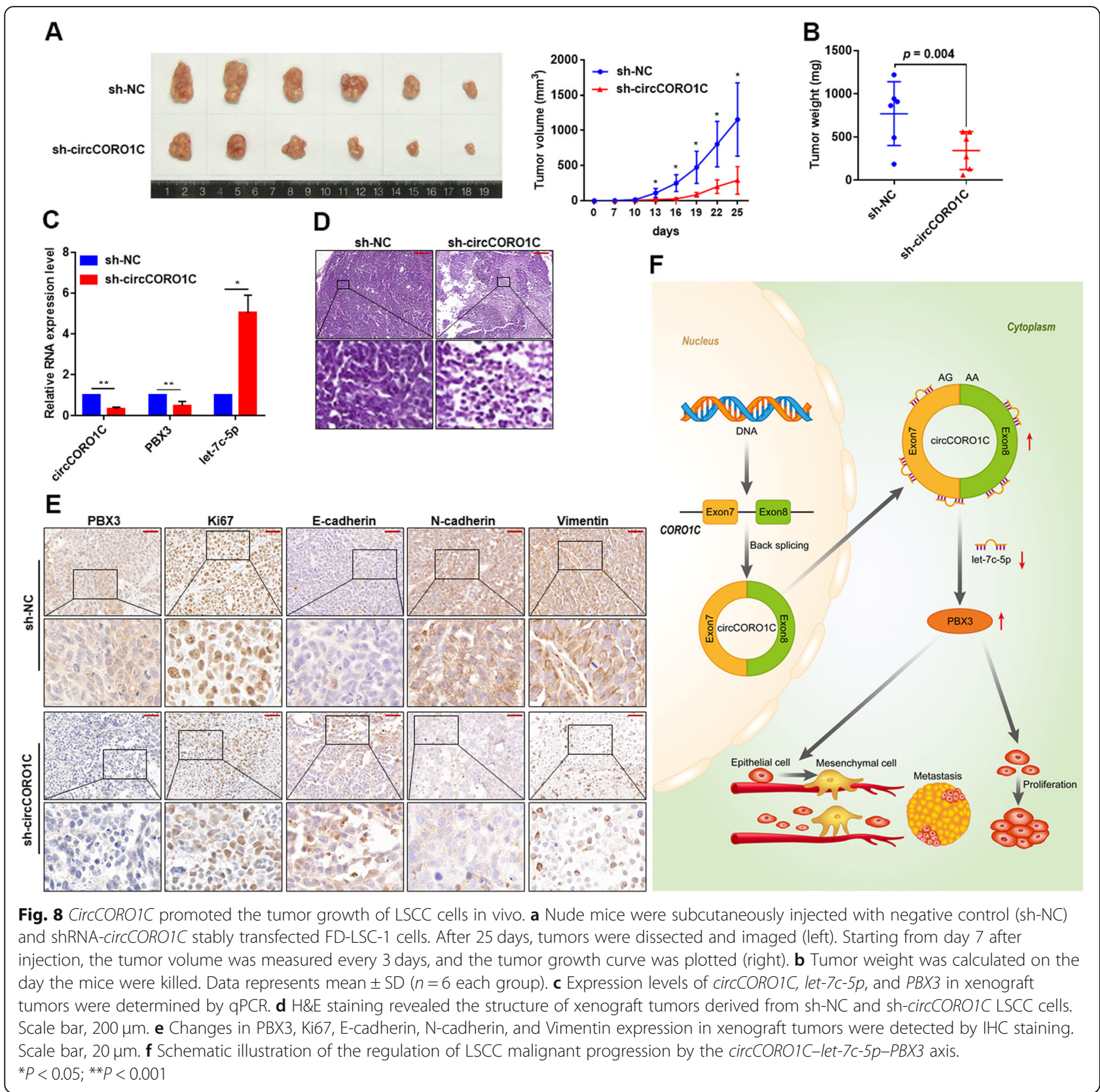

The CORO1C-encoded WD repeat protein family member regulates actin-dependent processes through Factin assembly [27]. Studies have shown that CORO1C promotes the metastases of breast cancer and lung squamous cell carcinoma $[28,29]$. Cheng et al. reported that CORO1C is highly expressed in gastric cancer tissues, and in vitro experiments demonstrated that CORO1C promotes the proliferation, migration, and invasion of gastric cancer cells [30]. However, it is unclear whether circRNA is formed by CORO1C, and the roles of CORO1C-formed circRNA in disease or normal physiological processes have not yet been reported. In this study, RNA sequencing data analysis and experiments demonstrated that circCORO1C, which was highly expressed in LSCC tissues, was composed of exons 7 and 8 of CORO1C. Treatment with actinomycin D showed that the half-life of $\operatorname{circCORO1C}$ was significantly longer than that of linear CORO1C RNA. RNase $\mathrm{R}$ has $3^{\prime}$ to $5^{\prime}$ exoribonuclease activity that digests all linear RNAs except circular RNA structures [31]. When treated with RNase $\mathrm{R}$, there is no significant change in circCORO1C level, proving that it has high stability as previously reported circRNA [32, 33]. Importantly, we found that the high expression of $\operatorname{circCORO1C}$ was positively correlated with advanced $\mathrm{T}$ stage, cervical lymph node metastasis, and clinical stage of LSCC, as well as 
poor prognosis in patients with LSCC, suggesting that circCORO1C may exert an important regulatory effect on the occurrence and development of LSCC.

RNA sequencing and bioinformatics analysis indicated that circRNA has an important regulatory effect in the occurrence and development of head and neck tumors [34]. Experimental studies further demonstrated that circHIPK3 promotes cell proliferation and invasion in nasopharyngeal carcinoma [35], while Hsa_circ_0005379 inhibits the cell migration, invasion, proliferation, and in vivo tumorigenesis of oral squamous cell carcinoma [36]. CircRNAs CDR1as and hsa_circ_0023028 promote the proliferation, migration, and invasion of LSCC cells $[37,38]$. Moreover, our previous studies found that the circRNA hg19_circ_0005033, which is highly expressed in LSCC stem cells, promotes proliferation, migration, invasion, and chemotherapy resistance [19]. There are very few LSCC cell lines available, among which FDLSC-1 and TU-177 are well-characterized [18, 39]. Our data showed that expression of circCORO1C in FD-LSC1 and TU-177 cells was higher than that in normal control cell lines. Therefore, we used these two cell lines to investigate the role of circCORO1C in LSCC cells. Consistent results showed that knockdown of circCORO1C inhibited cell proliferation, migration, invasion, and promoted apoptosis of LSCC, indicating that circCORO1C acts as an important oncogene to promote the malignant progression of LSCC.

Transcripts with the same miRNA binding site, such as circRNA, mRNA, and lncRNA, regulate the expression of each other by competitively binding miRNAs. These molecules form a complex and precise posttranscriptional regulatory network, namely the ceRNA network [40]. As an important member of the ceRNA network, circRNA is involved in the formation of the circRNA-miRNA-mRNA axis, which has regulatory functions in a variety of diseases and is the most reported mechanism of action of circRNA [41-43]. In this study, we found that $\operatorname{circCORO1C}$ was localized to the cytoplasm, suggesting that it functions as a ceRNA [44]. let$7 c-5 p$ has been demonstrated to have anti-tumor effects in malignant tumors including non-small cell lung cancer and liver cancer $[45,46]$. The combined bioinformatics prediction and transcriptomic analysis showed that


strated that let-7c-5p expression levels in LSCC were significantly lower than that in adjacent normal tissues, and overexpression of let-7c-5p inhibited cell proliferation, migration, and invasion in LSCC. The luciferase reporter assay and AGO2 RIP experiments demonstrated that let-


revealed that inhibition of $l e t-7 c-5 p$ reversed the inhibitory effect of knockdown of circCORO1C on LSCC malignant phenotypes. These findings indicated that
circCORO1C sponged let-7c-5p to exert tumorpromoting functions in LSCC cells.

PBX3 is highly expressed in a variety of cancer tissues, such as prostate and cervical cancer $[14,16]$. Han et al. demonstrated that PBX3 expression is a critical determinant for maintaining the characteristics of tumorinitiating cells in hepatocellular carcinoma [17]. In this study, we found that PBX3 expression was upregulated in LSCC tissues, and functional studies indicated that PBX3 promoted cell proliferation, migration, and invasion in LSCC. Our data revealed that $P B X 3$ was a direct target of let-7c-5p, and circCORO1C competitively bound to let-7c-5p and relieved the inhibitory effect of let-7c-5p on $P B X 3$ expression, thereby upregulating $P B X 3$ expression. We further confirmed that cir$c C O R O 1 C$ promoted the malignant progression of LSCC cells by upregulating $P B X 3$. EMT is the basis of tumor cell migration and invasion [47, 48], and PBX3 is an essential regulator of the EMT signaling network [13]. We observed that changes in the expression levels of circCORO1C or $P B X 3$ affected the expression of EMT markers, indicating that the circCORO1C-let-7c-5p$P B X 3$ axis promoted the migration and invasion of LSCC cells by regulating EMT.

Finally, we demonstrated that knockdown of circCORO1C inhibited the growth of LSCC cell xenograft tumors through preclinical models and verified the regulatory relationship of the circCORO1C-let-7c-5p-PBX3 axis in vivo. In future, exploring the upstream regulator of circCORO1C and developing non-invasive circCORO1C detection methods in LSCC and other HNSCC types will be of great significance in promoting clinical translation.

\section{Conclusions}

In summary, our data revealed that $\operatorname{circCORO1C}$ competitively binds let-7c-5p to eliminate its inhibitory effect on $P B X 3$, thereby promoting LSCC cell proliferation, migration, and invasion (Fig. 8f). High expression of circCORO1C is an important marker of poor prognosis for LSCC. These findings provide new insights into the occurrence and progression of LSCC and indicate the potential of $\operatorname{circCORO1C}$ as a biomarker and therapeutic target for LSCC.

\section{Supplementary information}

Supplementary information accompanies this paper at https://doi.org/10. 1186/s12943-020-01215-4.

Additional file 1: Table S1. Clinical features of 57 LSCC samples for RNA sequencing. Table S2. Clinical features of 107 LSCC samples for qPCR validation. Table S3. Differentially expressed circRNAs in LSCC tissues. Table S4. Differentially expressed miRNAs in LSCC tissues. Table S5. Differentially expressed mRNAs in LSCC tissues. Table S6. Primer sequences for RT-PCR and $\mathrm{qPCR}$ analysis. Table S7. Prediction of 
circCORO1C and miRNA interaction by seedVicious. Table S8. let-7c-5p target gene prediction by ENCORI. Table S9. Intersection of predicted let-7c-5p targets and upregulated mRNAs in LSCC tissues

Additional file 2: Figure S1. RNA sequencing and high-content screening reveals that cirCCORO1C affects the proliferation of LSCC cells. a Flowchart showing the steps for identifying functional circRNAs in LSCC. b Validation of circRNA expression in LSCC tissues by RT-PCR and Sanger sequencing. $\mathbf{c}$ High-content screening of circRNAs that affect the proliferation of LSCC cells. GFP-labeled FD-LSC-1 cells were transfected with siRNAs targeting the indicated circRNA. After $24 \mathrm{~h}$ transfection, cells were seeded into 96-well plates, and the cell number was counted at the indicated time points. Representative images (left) and fold change in cell count (right) are shown. Data are presented as the means \pm SD of three independent experiments. ${ }^{*} P<0.05$. Figure S2. FD-LSC-1 cells were transfected with let-7c-5p mimics or NC mimics for $48 \mathrm{~h}$, then RIP assay was performed using AGO2 antibody and circCORO1C levels were measured by $\mathrm{qPCR}$. ${ }^{*} P<0.001$

\section{Abbreviations}

LSCC: Laryngeal squamous cell carcinoma; circRNA: Circular RNA; ceRNA: Competing endogenous RNA; EMT: Epithelial-mesenchymal transition; ANM: Adjacent normal mucosa; qPCR: Quantitative real-time PCR FISH: Fluorescence in situ hybridization; EdU: 5-Ethynyl-2'-deoxyuridine; RIP: RNA immunoprecipitation; 3' UTR: 3'-untranslated region; HNSCC: Head and neck squamous cell carcinoma

\section{Acknowledgments}

We thank Prof. Tao Bai from the Department of Pathology, The First Hospital of Shanxi Medical University for pathological analysis of LSCC sections.

\section{Authors' contributions}

WG, WX, HZL, and CMA conceived the study and participated in the study design. YYW, YLZ, XWZ, FSD, YL, MN and HNG performed cell culture, colony formation, western blots, RIP, and flow cytometry assays. YYW, XWZ, LD, WQL, XTX, YFB and RH performed bioinformatics analysis, $\mathrm{HC}$, and pathological diagnosis. JBQ, YXQ, HLL, YZ, TY, and LL performed highcontent screening, EdU staining and luciferase reporter assays. YYW, YLZ, XWZ, FSD, YL, LSZ and HNG contributed FISH and qPCR experiments. YYW, WQL and YLZ performed xenograft experiments. YL, YFB and SXW contributed to the clinical samples collection, follow-up and clinical data analysis. YYW, YLZ and XWZ performed primer design and plasmid construction. YYW, YJG, WG, WX, HZL, and CMA analyzed the data, organized figures and wrote the manuscript. All authors read and approved the final manuscript.

\section{Funding}

This work was supported by the National Natural Science Foundation of China (grants: 81872210, 81802793, and 81802948), Postdoctoral Research Foundation of China (grants: 2016 M591412 and 2017 M610174), The Excellent talent science and technology innovation project of Shanxi Province (grants: 201605D211029, 201705D211018, and 201805D211007), Youth Science and Technology Research Fund of Shanxi Province (grants: 201901D211486, 201901D211490), Shanxi Province Scientific and Technological Achievements Transformation Guidance Foundation (grants: 201604D131002, 201604D132040, and 201804D131043), Youth Foundation of The First Hospital Affiliated with Shanxi Medical University (grant: YQ1503), Youth Top Talent Program Fund of Shanxi Province, Fund of Shanxi "1331" Project.

\section{Availability of data and materials}

RNA sequencing raw data and normalized results were deposited at GEO database (GSE127165, GSE133632). All data that support the findings of this study are available from the corresponding authors upon reasonable request.

\section{Ethics approval and consent to participate}

The clinical samples were obtained with the consent of patients and approved by The Medical Ethics Committee of The First Hospital of Shanxi Medical University. Informed consent per institutional guidelines was obtained from all patients who agreed to participate in this study. Animal experiments were conducted according to the Health Guide for the Care and Use of Laboratory Animals approved by the Animal Experimental Research Ethics Committee of Shanxi Medical University.

\section{Consent for publication}

Not applicable.

\section{Competing interests}

The authors declare that they have no competing interests.

\section{Author details}

${ }^{1}$ Shanxi Key Laboratory of Otorhinolaryngology Head and Neck Cancer Shanxi Medical University, Taiyuan 030001, Shanxi, People's Republic of China. ${ }^{2}$ Shanxi Province Clinical Medical Research Center for Precision Medicine of Head and Neck Cancer, The First Hospital of Shanxi Medical University, Taiyuan 030001, Shanxi, People's Republic of China. ${ }^{3}$ Department of Otolaryngology Head \& Neck Surgery, The First Hospital of Shanxi Medical University, Taiyuan 030001, Shanxi, People's Republic of China. ${ }^{4}$ Key Laboratory of Cellular Physiology, Ministry of Education, Shanxi Medical University, Taiyuan 030001, Shanxi, People's Republic of China. ${ }^{5}$ Department of Biochemistry \& Molecular Biology, Shanxi Medical University, Taiyuan 030001, Shanxi, People's Republic of China. 'Department of Otolaryngology Head \& Neck Surgery, The First Hospital, Jinzhou Medical University, Jinzhou 121001, Liaoning, People's Republic of China. ${ }^{7}$ Department of Pathology, Shanxi Cancer Hospital, Shanxi Medical University, Taiyuan 030013, Shanxi, People's Republic of China. ${ }^{8}$ Department of Otolaryngology Head \& Neck Surgery, Heping Hospital Affiliated to Changzhi Medical College, Changzhi 046000, Shanxi, People's Republic of China. ${ }^{9}$ Department of Cell Biology and Genetics, Basic Medical School of Shanxi Medical University, Taiyuan 030001, Shanxi, People's Republic of China. ${ }^{10}$ Department of Physiology, Shanxi Medical University, Taiyuan 030001, Shanxi, People's Republic of China. ${ }^{11}$ Department of Hepatobiliary and Pancreatic Surgery, The Second Affiliated Hospital, Zhejiang University School of Medicine, Hangzhou 310009 Zhejiang, People's Republic of China. ${ }^{12}$ Harry Perkins Institute of Medical Research, QEIl Medical Centre and Centre for Medical Research, the University of Western Australia, PO Box 7214, 6 Verdun Street, Nedlands, Perth, Western Australia 6009, Australia. ${ }^{13}$ General Hospital, Shenzhen University, Shenzhen 518055, Guangdong, People's Republic of China. ${ }^{14}$ Department of Head and Neck Surgery, Cancer Hospital, National Cancer Center, Chinese Academy of Medical Sciences \& Peking Union Medical College, Beijing 100021, People's Republic of China. ${ }^{15}$ Department of Otolaryngology Head \& Neck Surgery, Dalian Municipal Friendship Hospital, Dalian Medical University, Dalian 116100, Liaoning, People's Republic of China. ${ }^{16}$ Shandong Provincial ENT Hospital Affiliated to Shandong University, Jinan 250022, Shandong, People's Republic of China. ${ }^{17}$ Shandong Provincial Institute of Otolaryngology, Jinan 250022, Shandong, People's Republic of China. ${ }^{18}$ Key Laboratory of Otolaryngology, Ministry of Health, Shandong University, Jinan 250022, Shandong, People's Republic of China.

Received: 20 January 2020 Accepted: 11 May 2020 Published online: 02 June 2020

\section{References}

1. Bray F, Ferlay J, Soerjomataram I, Siegel RL, Torre LA, Jemal A. Global cancer statistics 2018: GLOBOCAN estimates of incidence and mortality worldwide for 36 cancers in 185 countries. CA Cancer J Clin. 2018;68:394-424.

2. Steuer CE, El-Deiry M, Parks JR, Higgins KA, Saba NF. An update on larynx cancer. CA Cancer J Clin. 2017;67:31-50.

3. Gao W, Zhang C, Li W, Li H, Sang J, Zhao Q, et al. Promoter methylationregulated miR-145-5p inhibits laryngeal squamous cell carcinoma progression by targeting FSCN1. Mol Ther. 2019;27:365-79.

4. Ang KK, Zhang Q, Rosenthal DI, Nguyen-Tan PF, Sherman EJ, Weber RS, et al. Randomized phase III trial of concurrent accelerated radiation plus cisplatin with or without cetuximab for stage III to IV head and neck carcinoma: RTOG 0522. J Clin Oncol. 2014:32:2940-50.

5. Siegel RL, Miller KD, Jemal A. Cancer statistics, 2016. CA Cancer J Clin. 2016; 66:7-30.

6. Kristensen LS, Andersen MS, Stagsted L, Ebbesen KK, Hansen TB, Kjems J. The biogenesis, biology and characterization of circular RNAs. Nat Rev Genet. 2019;20:675-91.

7. Zhao X, Cai Y, Xu J. Circular RNAs: biogenesis, mechanism, and function in human cancers. Int J Mol Sci. 2019;20:3926. 
8. Du WW, Zhang C, Yang W, Yong T, Awan FM, Yang BB. Identifying and characterizing circRNA-protein interaction. Theranostics. 2017;7:4183-91.

9. Yang $Y$, Gao X, Zhang M, et al. Novel role of FBXW7 circular RNA in repressing Glioma tumorigenesis. J Natl Cancer Inst. 2018;110:304-15.

10. Bach DH, Lee SK, Sood AK. Circular RNAs in Cancer. Mol Ther Nucleic Acids. 2019:16:118-29.

11. Yang $R$, Xing $L$, Zheng $X$, Sun $Y$, Wang $X$, Chen J. The circRNA circAGFG1 acts as a sponge of miR-195-5p to promote triple-negative breast cancer progression through regulating CCNE1 expression. Mol Cancer. 2019;18:4.

12. Zheng X, Chen L, Zhou Y, Wang Q, Zheng Z, Xu B, Wu C, Zhou Q, Hu W, Wu C, Jiang J. A novel protein encoded by a circular RNA circPPP1R12A promotes tumor pathogenesis and metastasis of colon cancer via hippoYAP signaling. Mol Cancer. 2019;18:47.

13. Lamprecht S, Kaller M, Schmidt EM, Blaj C, Schiergens TS, Engel J, Jung A, Hermeking H, Grünewald TGP, Kirchner T, Horst D. PBX3 is part of an EMT regulatory network and indicates poor outcome in colorectal Cancer. Clin Cancer Res. 2018;24:1974-86.

14. Ramberg H, Grytli HH, Nygård S, Wang W, Ögren O, Zhao S, Løvf M, Katz B, Skotheim Rl, Bjartell A, Eri LM, Berge V, Svindland A, Taskén KA. PBX3 is a putative biomarker of aggressive prostate cancer. Int J Cancer. 2016;139: 1810-20.

15. Li B, Zhang S, Shen H, Li C. MicroRNA-144-3p suppresses gastric cancer progression by inhibiting epithelial-to-mesenchymal transition through targeting PBX3. Biochem Biophys Res Commun. 2017;484:241-7.

16. Li H, Sun G, Liu C, Wang J, Jing R, Wang J, Zhao X, Xu X, Yang Y. PBX3 is associated with proliferation and poor prognosis in patients with cervical cancer. Onco Targets Ther. 2017;10:5685-94.

17. Han H, Du Y, Zhao W, Li S, Chen D, Zhang J, Liu J, Suo Z, Bian X, Xing B, Zhang Z. PBX3 is targeted by multiple miRNAs and is essential for liver tumour-initiating cells. Nat Commun. 2015:6:8271.

18. Wu CP, Zhou L, Gong HL, Du HD, Tian J, Sun S, Li JY. Establishment and characterization of a novel HPV-negative laryngeal squamous cell carcinoma cell line, FD-LSC-1, with missense and nonsense mutations of TP53 in the DNA-binding domain. Cancer Lett. 2014;342:92-103.

19. Wu Y, Zhang Y, Niu M, Shi Y, Liu H, Yang D, Li F, Lu Y, Bo Y, Zhang R, Li Z, Luo H, Cui J, Sang J, Xiang C, Gao W, Wen S. Whole-Transcriptome analysis of CD133+CD144+ Cancer stem cells derived from human laryngeal squamous cell carcinoma cells. Cell Physiol Biochem. 2018;47:1696-710.

20. Jeck WR, Sharpless NE. Detecting and characterizing circular RNAs. Nat Biotechnol. 2014;32:453-61.

21. Li Y, Chen B, Huang S. Identification of circRNAs for miRNA targets by Argonaute2 RNA Immunoprecipitation and luciferase screening assays. Methods Mol Biol. 1724:2018:209-18.

22. Yin $Y$, Long J, He Q, Li Y, Liao Y, He P, Zhu W. Emerging roles of circRNA in formation and progression of cancer. J Cancer. 2019;10:5015-21.

23. Arnaiz E, Sole C, Manterola L, Iparraguirre L, Otaegui D, Lawrie CH. CircRNAs and cancer: biomarkers and master regulators. Semin Cancer Biol. 2019;58:90-9.

24. Hsiao KY, Lin YC, Gupta SK, Chang N, Yen L, Sun HS, Tsai SJ. Noncoding effects of circular RNA CCDC66 promote Colon Cancer growth and metastasis. Cancer Res. 2017;77:2339-50

25. Fan Y, Xia X, Zhu Y, Diao W, Zhu X, Gao Z, Chen X. Circular RNA expression profile in laryngeal squamous cell carcinoma revealed by microarray. Cell Physiol Biochem. 2018;50:342-52.

26. Lu C, Shi X, Wang AY, Tao Y, Wang Z, Huang C, Qiao Y, Hu H, Liu L. RNASeq profiling of circular RNAs in human laryngeal squamous cell carcinomas. Mol Cancer. 2018;17:86.

27. Williamson RC, Cowell CA, Hammond CL, Bergen DJ, Roper JA, Feng $Y$, Rendall TC, Race PR, Bass MD. Coronin-1C and RCC2 guide mesenchymal migration by trafficking Rac1 and controlling GEF exposure. J Cell Sci. 2014; 127:4292-307.

28. Lim JP, Shyamasundar S, Gunaratne J, Scully OJ, Matsumoto K, Bay BH. YBX1 gene silencing inhibits migratory and invasive potential via CORO1C in breast cancer in vitro. BMC Cancer. 2017;17:201.

29. Mataki H, Enokida H, Chiyomaru T, Mizuno K, Matsushita R, Goto $Y$, Nishikawa R, Higashimoto I, Samukawa T, Nakagawa M, Inoue H, Seki N. Downregulation of the microRNA-1/133a cluster enhances cancer cell migration and invasion in lung-squamous cell carcinoma via regulation of Coronin1C. J Hum Genet. 2015;60:53-61.

30. Cheng X, Wang X, Wu Z, Tan S, Zhu T, Ding K. CORO1C expression is associated with poor survival rates in gastric cancer and promotes metastasis in vitro. FEBS Open Bio. 2019;9:1097-108.
31. Xiao MS, Wilusz JE. An improved method for circular RNA purification using RNase $R$ that efficiently removes linear RNAs containing G-quadruplexes or structured 3' ends. Nucleic Acids Res. 2019;47:8755-69.

32. Zhang Z, Yang T, Xiao J. Circular RNAs: promising biomarkers for human diseases. EBioMedicine. 2018;34:267-74.

33. D'Ambra E, Capauto D, Morlando M. Exploring the regulatory role of circular RNAs in neurodegenerative disorders. Int J Mol Sci. 2019;20:5477.

34. Wang WL, Yang Z, Zhang YJ, Lu P, Ni YK, Sun CF, Liu FY. Competing endogenous RNA analysis reveals the regulatory potency of circRNA_ 036186 in HNSCC. Int J Oncol. 2018;53:1529-43.

35. Ke Z, Xie F, Zheng C, Chen D. CircHIPK3 promotes proliferation and invasion in nasopharyngeal carcinoma by abrogating miR-4288-induced ELF3 inhibition. J Cell Physiol. 2019;234:1699-706.

36. Su W, Wang Y, Wang F, Sun S, Li M, Shen Y, Yang H. Hsa_circ_0005379 regulates malignant behavior of oral squamous cell carcinoma through the EGFR pathway. BMC Cancer. 2019;19:400.

37. Zhang J, Hu H, Zhao Y, Zhao Y. CDR1as is overexpressed in laryngeal squamous cell carcinoma to promote the tumour's progression via miR-7 signals. Cell Prolif. 2018:51:e12521.

38. Chen X, Su X, Zhu C, Zhou J. Knockdown of hsa_circ_0023028 inhibits cell proliferation, migration, and invasion in laryngeal cancer by sponging miR194-5p. Biosci Rep. 2019:39:BSR20190177.

39. Liu TJ, Zhang WW, Taylor DL, Roth JA, Goepfert H, Clayman GL. Growth suppression of human head and neck cancer cells by the introduction of a wild-type p53 gene via a recombinant adenovirus. Cancer Res. 1994;54: 3662-7.

40. Tay Y, Rinn J, Pandolfi PP. The multilayered complexity of ceRNA crosstalk and competition. Nature. 2014;505:344-52.

41. Bi W, Huang J, Nie C, Liu B, He G, Han J, Pang R, Ding Z, Xu J, Zhang J. CircRNA circRNA 102171 promotes papillary thyroid cancer progression through modulating CTNNBIP1-dependent activation of $\beta$-catenin pathway. J Exp Clin Cancer Res. 2018;37:275.

42. Li H, Xu JD, Fang XH, Zhu JN, Yang J, Pan R, Yuan SJ, Zeng N, Yang ZZ, Yang H, Wang XP, Duan JZ, Wang S, Luo JF, Wu SL, Shan ZX. Circular RNA circRNA_000203 aggravates cardiac hypertrophy via suppressing miR26b-5p and miR-140-3p binding to Gata4. Cardiovasc Res. 2019. https://doi.org/10. 1093/cvr/cvz215.

43. Sang $Y$, Chen B, Song X, Li Y, Liang Y, Han D, Zhang N, Zhang H, Liu Y, Chen T, Li C, Wang L, Zhao W, Yang Q. circRNA_0025202 regulates Tamoxifen sensitivity and tumor progression via regulating the miR-182-5p/ FOXO3a Axis in breast Cancer. Mol Ther. 2019;27:1638-52.

44. Liu F, Zhang H, Xie F, Tao D, Xiao X, Huang C, Wang M, Gu C, Zhang X, Jiang G. Hsa_circ_0001361 promotes bladder cancer invasion and metastasis through miR-491-5p/MMP9 axis. Oncogene. 2020;39:1696-709.

45. Zhao B, Han H, Chen J, Zhang Z, Li S, Fang F, Zheng Q, Ma Y, Zhang J, Wu $N$, Yang Y. MicroRNA let-7c inhibits migration and invasion of human nonsmall cell lung cancer by targeting ITGB3 and MAP 4K3. Cancer Lett. 2014; 342:43-51.

46. Huang Y, Xiang B, Liu Y, Wang Y, Kan H. LncRNA CDKN2B-AS1 promotes tumor growth and metastasis of human hepatocellular carcinoma by targeting let-7c-5p/NAP1L1 axis. Cancer Lett. 2018;437:56-66.

47. Mittal V. Epithelial Mesenchymal transition in tumor metastasis. Annu Rev Pathol. 2018;13:395-412.

48. Ribatti D. Epithelial-mesenchymal transition in morphogenesis, cancer progression and angiogenesis. Exp Cell Res. 2017;353:1-5.

\section{Publisher's Note}

Springer Nature remains neutral with regard to jurisdictional claims in published maps and institutional affiliations. 